\title{
Error estimates for a semi-implicit fully discrete finite element scheme for the mean curvature flow of graphs
}

\author{
KLAUS DECKELNICK \\ School of Mathematical Sciences, University of Sussex, Falmer, Brighton BN1 9QH, UK \\ AND \\ GERHARD DZIUK \\ Institut für Angewandte Mathematik, Universität Freiburg, Hermann Herder Str.10, \\ 79104 Freiburg, Germany
}

[Received 7 April 1999 and in revised form 13 April 2000]

\begin{abstract}
The efficient numerical simulation of the curvature-driven motion of interfaces is an important tool in several free- boundary problems. We treat the case of an interface which is given as a graph. The highly non-linear problem is discretized in space by piecewise linear finite elements. Although the problem is not in divergence form it can be written in a variational form which allows the use of the modern adaptive techniques of finite elements. The time discretization is carried out in a semiimplicit way such that in every time step a linear system with symmetric positive matrix has to be solved. Optimal error estimates are proved for the fully discrete problem under the assumption that the time-step size is bounded by the spatial grid size.
\end{abstract}

\section{Introduction}

The aim of this paper is to analyse a fully discrete finite element algorithm that approximates the mean curvature flow of graphs. A family $\left(\Gamma_{t}\right)_{t \in[0, T]}$ of $n$-dimensional surfaces in $\mathbb{R}^{n+1}$ is said to flow by mean curvature if the normal velocity $V$ of $\Gamma_{t}$ equals its mean curvature. We shall restrict our attention to two-dimensional surfaces which can be written as graphs over some bounded, smooth domain $\Omega \subset \mathbb{R}^{2}$, i.e. $\Gamma_{t}=\{(x, u(x, t)) \mid x \in \Omega\}$. Abbreviating by defining

$$
Q(u)=\sqrt{1+|\nabla u|^{2}},
$$

the downward pointing normal $v(u)$ to $\Gamma_{t}$ is given by

$$
v(u)=\left(\frac{\nabla u}{Q(u)},-\frac{1}{Q(u)}\right)
$$

while normal velocity and mean curvature with respect to $v(u)$ are calculated according to

$$
V=-\frac{u_{t}}{Q(u)}, \quad H=-\nabla \cdot \frac{\nabla u}{Q(u)} .
$$

Thus, the relation $V=H$ translates into the quasi-linear partial differential equation

$$
u_{t}-Q(u) \nabla \cdot \frac{\nabla u}{Q(u)}=0 \quad \text { in } \quad \Omega \times(0, T)
$$


to which we add the following boundary and initial conditions:

$$
\begin{array}{ll}
u(\cdot, t)=u_{0} & \text { on } \quad \partial \Omega \times(0, T) \\
u(\cdot, 0)=u_{0} & \text { in } \quad \Omega .
\end{array}
$$

Here, $u_{0}: \bar{\Omega} \rightarrow \mathbb{R}$ is a given smooth function.

If the mean curvature of $\partial \Omega$ is non-positive with respect to the exterior normal it was shown in [11] and [9] that (1.4), (1.5) has a global smooth solution. In general, however, smooth solutions exist only locally in time and the gradient can blow up at the boundary; cf. [13]. Results for Neumann-type boundary conditions can be found in $[1,2,9]$.

The differential equation (1.4) is not in divergence form and so we could expect that a numerical method like the finite element method, which is based on a variational formulation, might not be applicable here. But it is easy to see that the energy equality

$$
\int_{\Omega} \frac{u_{t}^{2}}{Q(u)}+\frac{\mathrm{d}}{\mathrm{d} t} \int_{\Omega} Q(u)=0
$$

holds for time-independent boundary values and this will lead us to a variational form of our problem. It will be possible to discretize the equation in space with piecewise linear finite elements and this means that the resulting scheme will be open to the use of modern adaptive methods. An error estimate for a semi-discrete scheme of this form has been proved in [8] for the isotropic and in [7] for the anisotropic mean curvature flow of graphs.

In this work we will derive a fully discrete scheme for (1.4) in which the discretization is such that it linearizes the problem by a semi-implicit choice of the time discretization. Under the assumption that

$$
\tau \leqslant \delta_{0} h,
$$

where $\tau$ is the time-step size and $h$ is the grid size in space, we will prove optimal asymptotic error estimates of the form

$$
\sup _{0 \leqslant m \leqslant M}\left\|u^{m}-u_{h}^{m}\right\|_{H^{1}(\Omega)} \leqslant c(\tau+h) .
$$

Here we used the common shorthand $v^{m}(x)=v(x, m \tau)$. The main idea for the proof will be a form of the energy identity (1.6) together with a superconvergence result for a non-linear Ritz Projection, which we call the minimal surface projection. An important fact will be the adequate use of geometric quantities. We complete the paper with some numerical tests which confirm the results of the error estimates precisely.

There are of course other approaches to studying motion by mean curvature which avoid the restriction that $\Gamma_{t}$ has to be a graph. Let us briefly review some of the corresponding work, concentrating on fully discrete algorithms and their numerical analysis.

Numerical schemes for the level set approach were introduced by Osher \& Sethian [14], while Walkington [15] proposes a finite element algorithm and studies its stability. Crandall \& Lions [4] introduced a monotone, convergent finite difference scheme which uses a regularization of the level set equation. An error estimate for this scheme is proved in [5].

A further possibility of approximating mean curvature motion is via the Allen-Cahn equation, a singularly perturbed parabolic equation. Convergence results for fully discrete algorithms are proved in [3] and [12]. 


\section{Discretization in space and time}

In order to derive our numerical algorithm we first rewrite (1.4) in the variational form

$$
\int_{\Omega} \frac{u_{t} \varphi}{Q(u)}+\int_{\Omega} \frac{\nabla u \cdot \nabla \varphi}{Q(u)}=0, \quad \varphi \in H_{0}^{1}(\Omega), \quad 0 \leqslant t \leqslant T
$$

together with (1.5). The fact, that (1.4) is not in divergence form is reflected in (2.1) by the appearance of a denominator in the first integral which complicates the analysis.

Next, let us consider a family $\mathcal{T}_{h}$ of triangulations of $\Omega$ (allowing the boundary elements to have a curved face) with maximum mesh size $h:=\max _{S \in \mathcal{T}_{h}} \operatorname{diam}(S)$ for which we assume the following regularity condition: there exists a constant $\kappa>0$ (which is independent of $h$ ) such that each $S \in \mathcal{T}_{h}$ is contained in a ball of radius $\kappa^{-1} h$ and contains a ball of radius $\kappa h$. The discrete space is chosen to be

$$
X_{h}:=\left\{v_{h} \in C^{0}(\bar{\Omega}) \mid v_{h} \text { is a linear polynomial on each } S \in \mathcal{T}_{h}\right\}
$$

where the isoparametric modification is used in curved elements; see [16]. We also set $X_{h 0}:=$ $X_{h} \cap H_{0}^{1}(\Omega)$. Furthermore, we denote by $\tau>0$ the time step and let $M=T / \tau$.

Recalling (2.1), it is natural to introduce the following scheme.

ALGORITHM 1 For $u_{h}^{0}=\hat{u}_{0 h}$ find $u_{h}^{m} \in X_{h}, \quad 0 \leqslant m \leqslant M-1$ such that $u_{h}^{m+1}-I_{h} u_{0} \in X_{h 0}$ and

$$
\frac{1}{\tau} \int_{\Omega} \frac{\left(u_{h}^{m+1}-u_{h}^{m}\right) \varphi_{h}}{Q\left(u_{h}^{m}\right)}+\int_{\Omega} \frac{\nabla u_{h}^{m+1} \cdot \nabla \varphi_{h}}{Q\left(u_{h}^{m}\right)}=0 \quad \text { for all } \quad \varphi_{h} \in X_{h 0} .
$$

Here, $I_{h}$ is the usual Lagrange interpolation operator and $\hat{u_{0 h}}$ denotes the minimal surface projection of $u_{0}$ which will be defined below. The above scheme is semi-implicit and requires the solution of a linear equation in each time step. Furthermore, the following stability estimate holds.

PROPOSITION 1 The solution $u_{h}^{m}, 0 \leqslant m \leqslant M$ of (2.2) satisfies for every $m \in\{1, \ldots, M\}$

$$
\begin{aligned}
& \tau \sum_{k=0}^{m-1} \int_{\Omega}\left|V_{h}^{k}\right|^{2} Q\left(u_{h}^{k}\right)+\sum_{k=0}^{m-1} \int_{\Omega}\left(Q\left(u_{h}^{k+1}\right)-Q\left(u_{h}^{k}\right)\right)^{2} \frac{1}{Q\left(u_{h}^{k}\right)} \\
& \quad+\frac{1}{2} \sum_{k=0}^{m-1} \int_{\Omega}\left|v\left(u_{h}^{k+1}\right)-v\left(u_{h}^{k}\right)\right|^{2} Q\left(u_{h}^{k+1}\right)+\int_{\Omega} Q\left(u_{h}^{m}\right)=\int_{\Omega} Q\left(u_{h}^{0}\right)
\end{aligned}
$$

where $V_{h}^{k}=-\frac{\left(u_{h}^{k+1}-u_{h}^{k}\right) / \tau}{Q\left(u_{h}^{k}\right)}$ is the discrete normal velocity.

Proof. See [8], Theorem 2.

Note that the above stability estimate is true without any restriction on the time step $\tau$. The purpose of the present work is to analyse the convergence of the scheme (2.2). To formulate our result we define $u^{m}:=u\left(\cdot, t_{m}\right), t_{m}=m \tau, 0 \leqslant m \leqslant M$. Then we have the following. 
THEOREM 1 Let $u$ be a smooth solution of (1.4), (1.5) and $u_{h}^{m}$ the solution of (2.2). Then there exists $\delta_{0}>0$ such that

$$
\begin{array}{r}
\sup _{0 \leqslant m \leqslant M}\left\|u^{m}-u_{h}^{m}\right\| \leqslant c\left(\tau+h^{2}|\log h|^{2}\right) \\
\sup _{0 \leqslant m \leqslant M}\left\|\nabla\left(u^{m}-u_{h}^{m}\right)\right\| \leqslant c(\tau+h)
\end{array}
$$

provided $\tau \leqslant \delta_{0} h$. Here, $\|\cdot\|$ denotes the $L^{2}(\Omega)$-norm.

The precise regularity assumptions on $u$ appear in Proposition 3 below. Although we formulated our error bounds in terms of the usual $L^{2}$-norms, we shall see that it is much more appropriate to work with the geometric quantities $v(u)$ and $Q(u)$. This is not entirely surprising as we deal with a geometric problem but it is interesting to see how the use of these quantities can simplify the analysis or even make it possible. Thus, rather than trying to estimate $\left\|\nabla\left(u^{m}-u_{h}^{m}\right)\right\|$ we shall focus on the quantity

$$
E^{m}:=\int_{\Omega}\left|v\left(u_{h}^{m}\right)-v\left(\hat{u}_{h}^{m}\right)\right|^{2} Q\left(u_{h}^{m}\right) .
$$

Here, $\hat{u}_{h}^{m}$ denotes the minimal surface projection of $u^{m}$ to be defined below. The main part of the proof of Theorem 1 then consists in deriving the estimate

$$
E^{m} \leqslant c\left(\tau^{2}+h^{4}|\log h|^{4}\right), \quad 0 \leqslant m \leqslant M .
$$

Note that there is superconvergence in $h$ (as we obtain $h^{4}|\log h|^{4}$ rather than $h^{2}$ ) and this effect will be crucial for our argument.

\section{Error estimates}

Let us start with some useful relations involving the geometric quantities $Q$ and $v$, which will be used frequently throughout the paper.

Proposition 2 Let $u$ and $v$ be in $H^{1, \infty}(\Omega)$. Then we have a.e. in $\Omega$ :

$$
\begin{aligned}
|Q(u)-Q(v)| & \leqslant Q(u) Q(v)|v(u)-v(v)| \\
|\nabla(v-u)| & \leqslant\left(1+\sup _{\Omega}|\nabla v|\right) Q(u)|v(u)-v(v)|, \\
\frac{\nabla v}{Q(u)} \cdot \nabla(v-u) & =Q(v)-Q(u)+\frac{(Q(v)-Q(u))^{2}}{2 Q(u)}+\frac{|\nabla(v-u)|^{2}}{2 Q(u)}, \\
|\nabla(u-v)|^{2} & =(Q(u)-Q(v))^{2}+|v(u)-v(v)|^{2} Q(u) Q(v) .
\end{aligned}
$$

Proof. The first relation follows from the fact that $\frac{-1}{Q(u)}, \frac{-1}{Q(v)}$ are the last components of $v(u), v(v)$ respectively. The second inequality is a consequence of

$$
\nabla v-\nabla u=Q(u)\left(\frac{\nabla v}{Q(v)}-\frac{\nabla u}{Q(u)}\right)+Q(u)\left(\frac{1}{Q(u)}-\frac{1}{Q(v)}\right) \nabla v .
$$

Finally, (3.3) and (3.4) follow from elementary calculations. 
Before we start with the proof of Theorem 1 we introduce an important tool for the analysis. For a given function $v \in H^{2}(\Omega) \cap H^{1, \infty}(\Omega)$ we define the minimal surface projection $\hat{v}_{h} \in X_{h}$ by the relations $I_{h} v-\hat{v}_{h} \in X_{h 0}$ and

$$
\int_{\Omega} \frac{\nabla v \cdot \nabla \varphi_{h}}{Q(v)}=\int_{\Omega} \frac{\nabla \hat{v}_{h} \cdot \nabla \varphi_{h}}{Q\left(\hat{v}_{h}\right)} \quad \text { for all } \quad \varphi_{h} \in X_{h 0} .
$$

Existence and uniqueness of $\hat{v}_{h}$, together with an error analysis, were established in [10]. For functions which also depend on time the following bounds are available.

Proposition 3 Let $u \in L^{\infty}\left((0, T) ; H^{4}(\Omega)\right) \cap L^{2}\left((0, T) ; H^{5}(\Omega)\right)$ with $u_{t} \in L^{\infty}\left((0, T) ; H^{2}(\Omega)\right)$ $\cap L^{2}\left((0, T) ; H^{3}(\Omega)\right), \nabla u_{t} \in L^{\infty}(\Omega \times(0, T)), u_{t t} \in L^{\infty}\left((0, T) ; L^{2}(\Omega)\right) \cap L^{2}\left((0, T) ; H^{1}(\Omega)\right)$. Then the error between $u$ and $\hat{u}_{h}$ can be estimated as follows:

$$
\begin{aligned}
\sup _{(0, T)}\left\|u-\hat{u}_{h}\right\|+h \sup _{(0, T)}\left\|\nabla\left(u-\hat{u}_{h}\right)\right\| & \leqslant c h^{2}, \\
\sup _{(0, T)}\left\|u-\hat{u}_{h}\right\|_{L^{\infty}(\Omega)}+h \sup _{(0, T)}\left\|\nabla\left(u-\hat{u}_{h}\right)\right\|_{L^{\infty}(\Omega)} & \leqslant c h^{2}|\log h|, \\
\sup _{(0, T)}\left\|u_{t}-\hat{u}_{h, t}\right\| & \leqslant c h^{2}|\log h|^{2}, \\
\sup _{(0, T)}\left\|\nabla\left(u_{t}-\hat{u}_{h, t}\right)\right\| & \leqslant c h .
\end{aligned}
$$

Proof. The proof is carried out in [6] for the case of zero boundary values of $u$, but it can be extended to inhomogeneous boundary values in a straightforward way. As for (3.8) we note that in [6] the weaker estimate $\int_{0}^{T}\left\|\nabla\left(u_{t}-\hat{u}_{h, t}\right)\right\|^{2} \leqslant c h^{2}$ is proved which was a consequence of (see [6], p. 202)

$$
\left\|\nabla\left(u_{t}-\hat{u}_{h, t}\right)\right\|^{2} \leqslant c h\left\|\nabla\left(u_{t}-\hat{u}_{h, t}\right)\right\|\left(\left\|\nabla u_{t}\right\|_{L^{\infty}}+\left\|u_{t}\right\|_{H^{2}}\right)+c h^{2}\left\|\nabla u_{t}\right\|_{L^{\infty}}\left\|u_{t}\right\|_{H^{2}} .
$$

However, our assumption $\nabla u_{t} \in L^{\infty}(\Omega \times(0, T))$ (which was not made in [6]) implies the stronger bound (3.8).

We shall keep the above assumptions concerning the regularity of $u$ throughout this work. Using the minimal surface projection and evaluating (2.1) at $t=t_{m}$ we obtain

$$
\int_{\Omega} \frac{u_{t}\left(\cdot, t_{m}\right) \varphi_{h}}{Q\left(u^{m}\right)}+\int_{\Omega} \frac{\nabla \hat{u}_{h}^{m} \cdot \nabla \varphi_{h}}{Q\left(\hat{u}_{h}^{m}\right)}=0 \quad \text { for all } \quad \varphi_{h} \in X_{h 0}
$$

and therefore

$$
\frac{1}{\tau} \int_{\Omega} \frac{\left(u^{m+1}-u^{m}\right) \varphi_{h}}{Q\left(u^{m}\right)}+\int_{\Omega} \frac{\nabla \hat{u}_{h}^{m+1} \cdot \nabla \varphi_{h}}{Q\left(\hat{u}_{h}^{m}\right)}=\frac{1}{\tau} \int_{\Omega} \frac{R^{m} \varphi_{h}}{Q\left(u^{m}\right)}+\int_{\Omega} \frac{\nabla\left(\hat{u}_{h}^{m+1}-\hat{u}_{h}^{m}\right) \cdot \nabla \varphi_{h}}{Q\left(\hat{u}_{h}^{m}\right)}
$$

where

$$
\left\|R^{m}\right\| \leqslant c \tau^{2}, \quad 0 \leqslant m \leqslant M-1 .
$$


Let us decompose the error $\mathrm{e}^{m}=u^{m}-u_{h}^{m}$ in the following way:

$$
\mathrm{e}^{m}=\left(u^{m}-\hat{u}_{h}^{m}\right)+\left(\hat{u}_{h}^{m}-u_{h}^{m}\right)=: \epsilon^{m}+\mathrm{e}_{h}^{m} .
$$

The difference between the continuous (3.9) and the discrete (2.2) equation evaluated at a discrete test function then gives

$$
\begin{aligned}
& \frac{1}{\tau} \int_{\Omega}\left(\frac{u^{m+1}-u^{m}}{Q\left(u^{m}\right)}-\frac{u_{h}^{m+1}-u_{h}^{m}}{Q\left(u_{h}^{m}\right)}\right) \varphi_{h}+\int_{\Omega}\left(\frac{\nabla \hat{u}_{h}^{m+1}}{Q\left(\hat{u}_{h}^{m}\right)}-\frac{\nabla u_{h}^{m+1}}{Q\left(u_{h}^{m}\right)}\right) \cdot \nabla \varphi_{h} \\
& \quad=\frac{1}{\tau} \int_{\Omega} \frac{R^{m} \varphi_{h}}{Q\left(u^{m}\right)}+\int_{\Omega} \frac{\nabla\left(\hat{u}_{h}^{m+1}-\hat{u}_{h}^{m}\right) \cdot \nabla \varphi_{h}}{Q\left(\hat{u}_{h}^{m}\right)}
\end{aligned}
$$

for all $\varphi_{h} \in X_{h 0}$. The weights $1 / Q$ within the integrals will be important for our reasoning and so we will use the following form of the above equation:

$$
\begin{aligned}
& \frac{1}{\tau} \int_{\Omega} \frac{\left(\mathrm{e}^{m+1}-\mathrm{e}^{m}\right) \varphi_{h}}{Q\left(u_{h}^{m}\right)}+\int_{\Omega}\left(\frac{\nabla \hat{u}_{h}^{m+1}}{Q\left(\hat{u}_{h}^{m}\right)}-\frac{\nabla u_{h}^{m+1}}{Q\left(u_{h}^{m}\right)}\right) \cdot \nabla \varphi_{h} \\
& \quad=\frac{1}{\tau} \int_{\Omega}\left(u^{m+1}-u^{m}\right)\left(\frac{1}{Q\left(u_{h}^{m}\right)}-\frac{1}{Q\left(u^{m}\right)}\right) \varphi_{h}+\frac{1}{\tau} \int_{\Omega} \frac{R^{m} \varphi_{h}}{Q\left(u^{m}\right)}+\int_{\Omega} \frac{\nabla\left(\hat{u}_{h}^{m+1}-\hat{u}_{h}^{m}\right) \cdot \nabla \varphi_{h}}{Q\left(\hat{u}_{h}^{m}\right)} .
\end{aligned}
$$

We choose

$$
\varphi_{h}=\left(\mathrm{e}_{h}^{m+1}-\mathrm{e}_{h}^{m}\right) / \tau=\left(\mathrm{e}^{m+1}-\mathrm{e}^{m}\right) / \tau-\left(\epsilon^{m+1}-\epsilon^{m}\right) / \tau
$$

as a test function and get

$$
\begin{aligned}
& \frac{1}{\tau^{2}} \int_{\Omega} \frac{\left(\mathrm{e}^{m+1}-\mathrm{e}^{m}\right)^{2}}{Q\left(u_{h}^{m}\right)}+\frac{1}{\tau} \int_{\Omega}\left(\frac{\nabla \hat{u}_{h}^{m+1}}{Q\left(\hat{u}_{h}^{m}\right)}-\frac{\nabla u_{h}^{m+1}}{Q\left(u_{h}^{m}\right)}\right) \cdot \nabla\left(\mathrm{e}_{h}^{m+1}-\mathrm{e}_{h}^{m}\right) \\
= & \frac{1}{\tau^{2}} \int_{\Omega} \frac{\left(\mathrm{e}^{m+1}-\mathrm{e}^{m}\right)\left(\epsilon^{m+1}-\epsilon^{m}\right)}{Q\left(u_{h}^{m}\right)}+\frac{1}{\tau} \int_{\Omega} \frac{\nabla\left(\hat{u}_{h}^{m+1}-\hat{u}_{h}^{m}\right) \nabla\left(\mathrm{e}_{h}^{m+1}-\mathrm{e}_{h}^{m}\right)}{Q\left(\hat{u}_{h}^{m}\right)} \\
& +\frac{1}{\tau^{2}} \int_{\Omega}\left(u^{m+1}-u^{m}\right)\left(\mathrm{e}_{h}^{m+1}-\mathrm{e}_{h}^{m}\right)\left(\frac{1}{Q\left(u_{h}^{m}\right)}-\frac{1}{Q\left(u^{m}\right)}\right)+\frac{1}{\tau^{2}} \int_{\Omega} \frac{R^{m}\left(\mathrm{e}_{h}^{m+1}-\mathrm{e}_{h}^{m}\right)}{Q\left(u^{m}\right)} .
\end{aligned}
$$

Before we start our estimates we prove a useful lemma.

Lemma 1 Suppose that $h^{-2} E^{m} \leqslant \gamma^{2}$ for some $\gamma \leqslant 1$. Then we have

$$
\begin{aligned}
\sup _{\Omega} Q\left(u_{h}^{m}\right) & \leqslant c, \\
\left\|\nabla \mathrm{e}_{h}^{m}\right\|^{2} & \leqslant c E^{m}, \\
\left\|\nabla \mathrm{e}_{h}^{m}\right\|_{L^{\infty}} & \leqslant c \gamma .
\end{aligned}
$$

Proof. We infer from (3.6) that $\left|\nabla \hat{u}_{h}^{m}\right|$ is bounded uniformly in $m$ and $h$. Thus, an inverse estimate 
together with (3.2) implies

$$
\begin{aligned}
\sup _{\Omega} Q\left(u_{h}^{m}\right) & \leqslant 1+\left\|\nabla u_{h}^{m}\right\|_{L^{\infty}} \leqslant c+\left\|\nabla\left(u_{h}^{m}-\hat{u}_{h}^{m}\right)\right\|_{L^{\infty}} \\
& \leqslant c+c h^{-1}\left\|\nabla\left(u_{h}^{m}-\hat{u}_{h}^{m}\right)\right\| \leqslant c+c h^{-1}\left(\int_{\Omega}\left|v\left(u_{h}^{m}\right)-v\left(\hat{u}_{h}^{m}\right)\right|^{2} Q\left(u_{h}^{m}\right)^{2}\right)^{\frac{1}{2}} \\
& \leqslant c+c \sqrt{\sup _{\Omega} Q\left(u_{h}^{m}\right)} h^{-1} \sqrt{E^{m}} \leqslant \frac{1}{2} \sup _{\Omega} Q\left(u_{h}^{m}\right)+c,
\end{aligned}
$$

which implies (3.13). To see (3.14), note that by (3.2) and (3.13)

$$
\left\|\nabla \mathrm{e}_{h}^{m}\right\|^{2}=\int_{\Omega}\left|\nabla\left(u_{h}^{m}-\hat{u}_{h}^{m}\right)\right|^{2} \leqslant c \int_{\Omega}\left|v\left(u_{h}^{m}\right)-v\left(\hat{u}_{h}^{m}\right)\right|^{2} Q\left(u_{h}^{m}\right)^{2} \leqslant c E^{m} .
$$

Finally, (3.15) again follows from an inverse estimate.

In what follows we shall assume that the condition

$$
h^{-2} E^{m} \leqslant \gamma^{2}
$$

is satisfied so that the results of Lemma 1 are available. We will justify this assumption at the end of our proof within an induction argument.

Let us now return to (3.12). The crucial term is the second one on the left-hand side which we shall bound from below by a suitable (discrete) time derivative minus some error terms. Since the corresponding calculations are quite technical we sketch the argument in the continuous case for the convenience of the reader. For two functions $v$ and $w$ which are smooth enough one can show the following inequality:

$$
\begin{aligned}
& \int_{\Omega}\left(\frac{\nabla v}{Q(v)}-\frac{\nabla w}{Q(w)}\right) \nabla\left(v_{t}-w_{t}\right)=\frac{\mathrm{d}}{\mathrm{d} t} \int_{\Omega} \frac{Q(v) Q(w)-(1+\nabla v \cdot \nabla w)}{Q(v)} \\
& \quad+\int_{\Omega} \nabla v_{t} \cdot\left(\frac{\nabla w}{Q(v)}-\frac{\nabla w}{Q(w)}+\frac{\nabla v}{Q(v)}-\frac{1+\nabla v \cdot \nabla w}{Q(v)^{2}} \frac{\nabla v}{Q(v)}\right) \\
& \geqslant \frac{1}{2} \frac{\mathrm{d}}{\mathrm{d} t} \int_{\Omega}|v(v)-v(w)|^{2} Q(w)-\int_{\Omega}\left|\nabla v_{t}\right||v(v)-v(w)|^{2} Q(w) .
\end{aligned}
$$

This relation translates into the time-discrete setting as follows.

LEMMA 2 For sufficiently small $\gamma$ we have

$$
\begin{aligned}
& \frac{1}{\tau} \int_{\Omega}\left(\frac{\nabla \hat{u}_{h}^{m+1}}{Q\left(\hat{u}_{h}^{m}\right)}-\frac{\nabla u_{h}^{m+1}}{Q\left(u_{h}^{m}\right)}\right) \cdot \nabla\left(\mathrm{e}_{h}^{m+1}-\mathrm{e}_{h}^{m}\right) \geqslant \frac{1}{2 \tau}\left(E^{m+1}-E^{m}\right) \\
& \quad+\frac{1}{4 \tau} \int_{\Omega} \frac{\left|\nabla\left(\mathrm{e}_{h}^{m+1}-\mathrm{e}_{h}^{m}\right)\right|^{2}}{Q\left(\hat{u}_{h}^{m}\right)}-c\left(E^{m}+E^{m+1}\right)-c \tau^{2} .
\end{aligned}
$$

Proof. Let us denote the integral on the left-hand side by L. Clearly,

$$
L=\frac{1}{\tau} \int_{\Omega}\left(\frac{\nabla \hat{u}_{h}^{m+1}}{Q\left(\hat{u}_{h}^{m}\right)}-\frac{\nabla u_{h}^{m+1}}{Q\left(u_{h}^{m}\right)}\right) \cdot\left(\nabla\left(\hat{u}_{h}^{m+1}-\hat{u}_{h}^{m}\right)-\nabla\left(u_{h}^{m+1}-u_{h}^{m}\right)\right) .
$$


From (3.3) and the trivial identity

$$
\frac{1}{2}|v(u)-v(v)|^{2}=1-\frac{1+\nabla u \cdot \nabla v}{Q(u) Q(v)}
$$

we infer

$$
\begin{aligned}
\tau L= & \int_{\Omega} Q\left(\hat{u}_{h}^{m+1}\right)-Q\left(\hat{u}_{h}^{m}\right)+Q\left(u_{h}^{m+1}\right)-Q\left(u_{h}^{m}\right) \\
& +\int_{\Omega} \frac{\left(Q\left(\hat{u}_{h}^{m+1}\right)-Q\left(\hat{u}_{h}^{m}\right)\right)^{2}}{2 Q\left(\hat{u}_{h}^{m}\right)}+\frac{\left(Q\left(u_{h}^{m+1}\right)-Q\left(u_{h}^{m}\right)\right)^{2}}{2 Q\left(u_{h}^{m}\right)} \\
& +\int_{\Omega} \frac{\left|\nabla\left(\hat{u}_{h}^{m+1}-\hat{u}_{h}^{m}\right)\right|^{2}}{2 Q\left(\hat{u}_{h}^{m}\right)}+\frac{\left|\nabla\left(u_{h}^{m+1}-u_{h}^{m}\right)\right|^{2}}{2 Q\left(u_{h}^{m}\right)} \\
& -\int_{\Omega}\left(\frac{1}{Q\left(\hat{u}_{h}^{m}\right)}+\frac{1}{Q\left(u_{h}^{m}\right)}\right) \nabla \hat{u}_{h}^{m+1} \cdot \nabla u_{h}^{m+1}+\int_{\Omega} \frac{\nabla \hat{u}_{h}^{m+1} \cdot \nabla u_{h}^{m}}{Q\left(\hat{u}_{h}^{m}\right)}+\frac{\nabla u_{h}^{m+1} \cdot \nabla \hat{u}_{h}^{m}}{Q\left(u_{h}^{m}\right)} \\
= & \int_{\Omega} \frac{1}{2}\left|v\left(\hat{u}_{h}^{m+1}\right)-v\left(u_{h}^{m+1}\right)\right|^{2} Q\left(u_{h}^{m+1}\right)-\frac{1}{2}\left|v\left(\hat{u}_{h}^{m}\right)-v\left(u_{h}^{m}\right)\right|^{2} Q\left(u_{h}^{m}\right) \\
& +\int_{\Omega} \frac{\left(Q\left(\hat{u}_{h}^{m+1}\right)-Q\left(\hat{u}_{h}^{m}\right)\right)^{2}}{2 Q\left(\hat{u}_{h}^{m}\right)}+\frac{\left(Q\left(u_{h}^{m+1}\right)-Q\left(u_{h}^{m}\right)\right)^{2}}{2 Q\left(u_{h}^{m}\right)} \\
& +\int_{\Omega} \frac{\left|\nabla\left(\hat{u}_{h}^{m+1}-\hat{u}_{h}^{m}\right)\right|^{2}}{2 Q\left(\hat{u}_{h}^{m}\right)}+\frac{\left|\nabla\left(u_{h}^{m+1}-u_{h}^{m}\right)\right|^{2}}{2 Q\left(u_{h}^{m}\right)}+\int_{\Omega} S^{m}
\end{aligned}
$$

where

$$
\begin{aligned}
S^{m}= & Q\left(\hat{u}_{h}^{m+1}\right)-Q\left(\hat{u}_{h}^{m}\right)+\frac{1+\nabla \hat{u}_{h}^{m+1} \cdot \nabla u_{h}^{m+1}}{Q\left(\hat{u}_{h}^{m+1}\right)}-\frac{1+\nabla \hat{u}_{h}^{m} \cdot \nabla u_{h}^{m}}{Q\left(\hat{u}_{h}^{m}\right)} \\
& -\left(\frac{1}{Q\left(\hat{u}_{h}^{m}\right)}+\frac{1}{Q\left(u_{h}^{m}\right)}\right) \nabla \hat{u}_{h}^{m+1} \cdot \nabla u_{h}^{m+1}+\frac{\nabla \hat{u}_{h}^{m+1} \cdot \nabla u_{h}^{m}}{Q\left(\hat{u}_{h}^{m}\right)}+\frac{\nabla u_{h}^{m+1} \cdot \nabla \hat{u}_{h}^{m}}{Q\left(u_{h}^{m}\right)} \\
= & Q\left(\hat{u}_{h}^{m+1}\right)-Q\left(\hat{u}_{h}^{m}\right)+\left(1+\nabla \hat{u}_{h}^{m+1} \cdot \nabla u_{h}^{m+1}\right)\left(\frac{1}{Q\left(\hat{u}_{h}^{m+1}\right)}-\frac{1}{Q\left(u_{h}^{m}\right)}\right) \\
& -\left(1+\nabla u_{h}^{m+1} \cdot \nabla \hat{u}_{h}^{m}\right)\left(\frac{1}{Q\left(\hat{u}_{h}^{m}\right)}-\frac{1}{Q\left(u_{h}^{m}\right)}\right)-\frac{\nabla\left(\hat{u}_{h}^{m+1}-\hat{u}_{h}^{m}\right) \cdot \nabla\left(u_{h}^{m+1}-u_{h}^{m}\right)}{Q\left(\hat{u}_{h}^{m}\right)} .
\end{aligned}
$$


Using again (3.17) this term can be rewritten as follows:

$$
\begin{aligned}
S^{m}= & \left(1-\frac{1}{2}\left|v\left(\hat{u}_{h}^{m+1}\right)-v\left(u_{h}^{m+1}\right)\right|^{2}\right) Q\left(u_{h}^{m+1}\right)\left(1-\frac{Q\left(\hat{u}_{h}^{m+1}\right)}{Q\left(u_{h}^{m}\right)}\right) \\
& -\left(1-\frac{1}{2}\left|v\left(u_{h}^{m+1}\right)-v\left(\hat{u}_{h}^{m}\right)\right|^{2}\right) Q\left(u_{h}^{m+1}\right)\left(1-\frac{Q\left(\hat{u}_{h}^{m}\right)}{Q\left(u_{h}^{m}\right)}\right) \\
& +Q\left(\hat{u}_{h}^{m+1}\right)-Q\left(\hat{u}_{h}^{m}\right)-\frac{\nabla\left(\hat{u}_{h}^{m+1}-\hat{u}_{h}^{m}\right) \cdot \nabla\left(u_{h}^{m+1}-u_{h}^{m}\right)}{Q\left(\hat{u}_{h}^{m}\right)} \\
= & -\frac{1}{2}\left|v\left(\hat{u}_{h}^{m+1}\right)-v\left(u_{h}^{m+1}\right)\right|^{2} Q\left(u_{h}^{m+1}\right)\left(1-\frac{Q\left(\hat{u}_{h}^{m+1}\right)}{Q\left(u_{h}^{m}\right)}\right) \\
& +\frac{1}{2}\left|v\left(u_{h}^{m+1}\right)-v\left(\hat{u}_{h}^{m}\right)\right|^{2} Q\left(u_{h}^{m+1}\right)\left(1-\frac{Q\left(\hat{u}_{h}^{m}\right)}{Q\left(u_{h}^{m}\right)}\right) \\
& -\frac{\left(Q\left(\hat{u}_{h}^{m+1}\right)-Q\left(\hat{u}_{h}^{m}\right)\right)\left(Q\left(u_{h}^{m+1}\right)-Q\left(u_{h}^{m}\right)\right)}{Q\left(u_{h}^{m}\right)}-\frac{\nabla\left(\hat{u}_{h}^{m+1}-\hat{u}_{h}^{m}\right) \cdot \nabla\left(u_{h}^{m+1}-u_{h}^{m}\right)}{Q\left(\hat{u}_{h}^{m}\right)} .
\end{aligned}
$$

Inserting the above expression into (3.18) we obtain

$$
\begin{aligned}
2 \tau L= & \int_{\Omega}\left|v\left(\hat{u}_{h}^{m+1}\right)-v\left(u_{h}^{m+1}\right)\right|^{2} Q\left(u_{h}^{m+1}\right)-\int_{\Omega}\left|v\left(\hat{u}_{h}^{m}\right)-v\left(u_{h}^{m}\right)\right|^{2} Q\left(u_{h}^{m}\right) \\
& +\int_{\Omega} \frac{\left(Q\left(\hat{u}_{h}^{m+1}\right)-Q\left(\hat{u}_{h}^{m}\right)\right)^{2}}{Q\left(\hat{u}_{h}^{m}\right)}+\int_{\Omega} \frac{\left(Q\left(u_{h}^{m+1}\right)-Q\left(u_{h}^{m}\right)\right)^{2}}{Q\left(u_{h}^{m}\right)} \\
& +\int_{\Omega} \frac{\left|\nabla\left(\hat{u}_{h}^{m+1}-\hat{u}_{h}^{m}\right)\right|^{2}}{Q\left(\hat{u}_{h}^{m}\right)}+\int_{\Omega} \frac{\left|\nabla\left(u_{h}^{m+1}-u_{h}^{m}\right)\right|^{2}}{Q\left(u_{h}^{m}\right)} \\
& +\int_{\Omega}\left|v\left(\hat{u}_{h}^{m+1}\right)-v\left(u_{h}^{m+1}\right)\right|^{2} Q\left(u_{h}^{m+1}\right)\left(\frac{Q\left(\hat{u}_{h}^{m+1}\right)}{Q\left(u_{h}^{m}\right)}-1\right) \\
& +\int_{\Omega}\left|v\left(\hat{u}_{h}^{m}\right)-v\left(u_{h}^{m+1}\right)\right|^{2} Q\left(u_{h}^{m+1}\right)\left(1-\frac{Q\left(\hat{u}_{h}^{m}\right)}{Q\left(u_{h}^{m}\right)}\right) \\
& -2 \int_{\Omega} \frac{\left(Q\left(\hat{u}_{h}^{m+1}\right)-Q\left(\hat{u}_{h}^{m}\right)\right)\left(Q\left(u_{h}^{m+1}\right)-Q\left(u_{h}^{m}\right)\right)}{Q\left(u_{h}^{m}\right)} \\
& -2 \int_{\Omega} \frac{\nabla\left(\hat{u}_{h}^{m+1}-\hat{u}_{h}^{m}\right) \cdot \nabla\left(u_{h}^{m+1}-u_{h}^{m}\right)}{Q\left(\hat{u}_{h}^{m}\right)}
\end{aligned}
$$




$$
\begin{aligned}
= & \int_{\Omega}\left|v\left(\hat{u}_{h}^{m+1}\right)-v\left(u_{h}^{m+1}\right)\right|^{2} Q\left(u_{h}^{m+1}\right)-\int_{\Omega}\left|v\left(\hat{u}_{h}^{m}\right)-v\left(u_{h}^{m}\right)\right|^{2} Q\left(u_{h}^{m}\right) \\
& +\int_{\Omega} \frac{\left(\left(Q\left(\hat{u}_{h}^{m+1}\right)-Q\left(u_{h}^{m+1}\right)\right)-\left(Q\left(\hat{u}_{h}^{m}\right)-Q\left(u_{h}^{m}\right)\right)\right)^{2}}{Q\left(u_{h}^{m}\right)}+\int_{\Omega} \frac{\left|\nabla\left(\mathrm{e}_{h}^{m+1}-\mathrm{e}_{h}^{m}\right)\right|^{2}}{Q\left(\hat{u}_{h}^{m}\right)} \\
& +\int_{\Omega}\left(Q\left(\hat{u}_{h}^{m+1}\right)-Q\left(\hat{u}_{h}^{m}\right)\right)^{2}\left(\frac{1}{Q\left(\hat{u}_{h}^{m}\right)}-\frac{1}{Q\left(u_{h}^{m}\right)}\right) \\
& +\int_{\Omega}\left|\nabla\left(u_{h}^{m+1}-u_{h}^{m}\right)\right|^{2}\left(\frac{1}{Q\left(u_{h}^{m}\right)}-\frac{1}{Q\left(\hat{u}_{h}^{m}\right)}\right) \\
& +\int_{\Omega}\left|v\left(\hat{u}_{h}^{m+1}\right)-v\left(u_{h}^{m+1}\right)\right|^{2} Q\left(u_{h}^{m+1}\right)\left(\frac{Q\left(\hat{u}_{h}^{m+1}\right)}{Q\left(u_{h}^{m}\right)}-1\right) \\
& +\int_{\Omega}\left|v\left(\hat{u}_{h}^{m}\right)-v\left(u_{h}^{m+1}\right)\right|^{2} Q\left(u_{h}^{m+1}\right)\left(1-\frac{Q\left(\hat{u}_{h}^{m}\right)}{Q\left(u_{h}^{m}\right)}\right) \\
= & \int_{\Omega}\left|v\left(\hat{u}_{h}^{m+1}\right)-v\left(u_{h}^{m+1}\right)\right|^{2} Q\left(u_{h}^{m+1}\right)-\int_{\Omega}\left|v\left(\hat{u}_{h}^{m}\right)-v\left(u_{h}^{m}\right)\right|^{2} Q\left(u_{h}^{m}\right) \\
& +\int_{\Omega} \frac{\left(\left(Q\left(\hat{u}_{h}^{m+1}\right)-Q\left(u_{h}^{m+1}\right)\right)-\left(Q\left(\hat{u}_{h}^{m}\right)-Q\left(u_{h}^{m}\right)\right)\right)^{2}}{Q\left(u_{h}^{m}\right)}+\int_{\Omega} \frac{\left|\nabla\left(\mathrm{e}_{h}^{m+1}-\mathrm{e}_{h}^{m}\right)\right|^{2}}{Q\left(\hat{u}_{h}^{m}\right)} \\
& +\int_{\Omega}\left|v\left(u_{h}^{m+1}\right)-v\left(u_{h}^{m}\right)\right|^{2} Q\left(u_{h}^{m+1}\right)\left(1-\frac{Q\left(u_{h}^{m}\right)}{Q\left(\hat{u}_{h}^{m}\right)}\right) \\
& +\int_{\Omega}\left(\left(Q\left(u_{h}^{m+1}\right)-Q\left(u_{h}^{m}\right)\right)^{2}-\left(Q\left(\hat{u}_{h}^{m+1}\right)-Q\left(\hat{u}_{h}^{m}\right)\right)^{2}\right)\left(\frac{1}{Q\left(u_{h}^{m}\right)}-\frac{1}{Q\left(\hat{u}_{h}^{m}\right)}\right) \\
& +\int_{\Omega}\left|v\left(\hat{u}_{h}^{m+1}\right)-v\left(u_{h}^{m+1}\right)\right|^{2} Q\left(u_{h}^{m+1}\right)\left(\frac{Q\left(\hat{u}_{h}^{m+1}\right)}{Q\left(u_{h}^{m}\right)}-1\right) \\
& +\int_{\Omega}\left|v\left(\hat{u}_{h}^{m}\right)-v\left(u_{h}^{m+1}\right)\right|^{2} Q\left(u_{h}^{m+1}\right)\left(1-\frac{Q\left(\hat{u}_{h}^{m}\right)}{Q\left(u_{h}^{m}\right)}\right), \\
&
\end{aligned}
$$

where we used (3.4) in order to rewrite $\left|\nabla\left(u_{h}^{m+1}-u_{h}^{m}\right)\right|^{2}$. Recalling the definition (2.4) of $E^{m}$ we finally arrive at

$$
\begin{aligned}
2 \tau L= & E^{m+1}-E^{m}+\int_{\Omega} \frac{\left(\left(Q\left(\hat{u}_{h}^{m+1}\right)-Q\left(u_{h}^{m+1}\right)\right)-\left(Q\left(\hat{u}_{h}^{m}\right)-Q\left(u_{h}^{m}\right)\right)\right)^{2}}{Q\left(u_{h}^{m}\right)} \\
& +\int_{\Omega} \frac{\left|\nabla\left(\mathrm{e}_{h}^{m+1}-\mathrm{e}_{h}^{m}\right)\right|^{2}}{Q\left(\hat{u}_{h}^{m}\right)}+\sum_{i=1}^{4} S_{i},
\end{aligned}
$$

where $S_{1}, \ldots, S_{4}$ are the last four terms in the equation above. We are going to examine these integrals separately. To begin, note that (3.1) and (3.13) imply

$$
\left|1-\frac{Q\left(u_{h}^{m}\right)}{Q\left(\hat{u}_{h}^{m}\right)}\right|=\frac{1}{Q\left(\hat{u}_{h}^{m}\right)}\left|Q\left(\hat{u}_{h}^{m}\right)-Q\left(u_{h}^{m}\right)\right| \leqslant Q\left(u_{h}^{m}\right)\left|v\left(\hat{u}_{h}^{m}\right)-v\left(u_{h}^{m}\right)\right| \leqslant c\left|v\left(\hat{u}_{h}^{m}\right)-v\left(u_{h}^{m}\right)\right|,
$$


so that

$$
\left|S_{1}\right| \leqslant c \int_{\Omega}\left|v\left(u_{h}^{m+1}\right)-v\left(u_{h}^{m}\right)\right|^{2}\left|v\left(u_{h}^{m}\right)-v\left(\hat{u}_{h}^{m}\right)\right| Q\left(u_{h}^{m+1}\right) .
$$

We define

$$
q_{h}^{m}:=\left(Q\left(u_{h}^{m+1}\right)-Q\left(\hat{u}_{h}^{m+1}\right)\right)-\left(Q\left(u_{h}^{m}\right)-Q\left(\hat{u}_{h}^{m}\right)\right) .
$$

The estimate (3.13) together with the boundedness of $\left|\nabla \hat{u}_{h}^{m}\right|$ yields

$$
Q\left(u_{h}^{m+1}\right) \leqslant c+\left|q_{h}^{m}\right| .
$$

Using this in (3.20) we get for small enough $\delta>0$

$$
\begin{aligned}
\left|S_{1}\right| & \leqslant c \int_{\Omega}\left|v\left(u_{h}^{m+1}\right)-v\left(u_{h}^{m}\right)\right|^{2}\left|v\left(u_{h}^{m}\right)-v\left(\hat{u}_{h}^{m}\right)\right|\left(1+\left|q_{h}^{m}\right|\right) \\
& \leqslant \delta \int_{\Omega} \frac{\left|q_{h}^{m}\right|^{2}}{Q\left(u_{h}^{m}\right)}+\frac{c}{\delta} \int_{\Omega}\left|v\left(u_{h}^{m+1}\right)-v\left(u_{h}^{m}\right)\right|^{2}\left|v\left(u_{h}^{m}\right)-v\left(\hat{u}_{h}^{m}\right)\right| .
\end{aligned}
$$

Here we used again (3.13) as well as the fact that $|v(u)| \leqslant 1$. Next we observe that (3.4) implies in view of $Q(u), Q(v) \geqslant 1$

$$
|v(u)-v(v)| \leqslant|\nabla(u-v)| \quad \text { a.e. in } \quad \Omega
$$

for $u, v \in H^{1, \infty}(\Omega)$. In particular, we derive

$$
\begin{aligned}
\left|v\left(u_{h}^{m+1}\right)-v\left(u_{h}^{m}\right)\right| & \leqslant\left|\nabla\left(u_{h}^{m+1}-u_{h}^{m}\right)\right| \leqslant\left|\nabla\left(\mathrm{e}_{h}^{m+1}-\mathrm{e}_{h}^{m}\right)\right|+\left|\nabla\left(\hat{u}_{h}^{m+1}-\hat{u}_{h}^{m}\right)\right| \\
& \leqslant\left|\nabla\left(\mathrm{e}_{h}^{m+1}-\mathrm{e}_{h}^{m}\right)\right|+c \tau \sup _{(0, T)}\left\|\nabla \hat{u}_{h, t}\right\|_{L^{\infty}} \\
& \leqslant\left|\nabla\left(\mathrm{e}_{h}^{m+1}-\mathrm{e}_{h}^{m}\right)\right|+c \tau
\end{aligned}
$$

which follows from (3.8) together with an inverse estimate. We insert (3.23) into (3.21) and use (3.22), (3.15) to find

$$
\begin{aligned}
\left|S_{1}\right| \leqslant & \delta \int_{\Omega} \frac{\left|q_{h}^{m}\right|^{2}}{Q\left(u_{h}^{m}\right)}+\frac{c}{\delta}\left(\int_{\Omega}\left|\nabla\left(\mathrm{e}_{h}^{m+1}-\mathrm{e}_{h}^{m}\right)\right|^{2}\left|\nabla \mathrm{e}_{h}^{m}\right|+\tau^{2} \int_{\Omega}\left|v\left(u_{h}^{m}\right)-v\left(\hat{u}_{h}^{m}\right)\right|\right) \\
\leqslant & \delta \int_{\Omega} \frac{\left|q_{h}^{m}\right|^{2}}{Q\left(u_{h}^{m}\right)}+\frac{c}{\delta}\left(\left\|\nabla \mathrm{e}_{h}^{m}\right\|_{L^{\infty}} \int_{\Omega} \frac{\left|\nabla\left(\mathrm{e}_{h}^{m+1}-\mathrm{e}_{h}^{m}\right)\right|^{2}}{Q\left(\hat{u}_{h}^{m}\right)}+\tau^{2} \int_{\Omega}\left|v\left(u_{h}^{m}\right)-v\left(\hat{u}_{h}^{m}\right)\right|\right) \\
\leqslant & \delta \int_{\Omega} \frac{\left(\left(Q\left(\hat{u}_{h}^{m+1}\right)-Q\left(u_{h}^{m+1}\right)\right)-\left(Q\left(\hat{u}_{h}^{m}\right)-Q\left(u_{h}^{m}\right)\right)\right)^{2}}{Q\left(u_{h}^{m}\right)} \\
& +\frac{c}{\delta} \gamma \int_{\Omega} \frac{\left|\nabla\left(\mathrm{e}_{h}^{m+1}-\mathrm{e}_{h}^{m}\right)\right|^{2}}{Q\left(\hat{u}_{h}^{m}\right)}+\frac{c}{\delta}\left(\tau^{3}+\tau E^{m}\right),
\end{aligned}
$$

where the last estimate follows from Hölder's inequality and the definitions of $q_{h}^{m}$ and $E^{m}$. Next, letting $a=Q\left(u_{h}^{m+1}\right)-Q\left(u_{h}^{m}\right)$ and $b=Q\left(\hat{u}_{h}^{m+1}\right)-Q\left(\hat{u}_{h}^{m}\right)$ we have $a-b=q_{h}^{m}\left(q_{h}^{m}\right.$ as above $)$ and

$$
\left|a^{2}-b^{2}\right|=\left|(a-b)^{2}+2 b(a-b)\right| \leqslant 2|a-b|^{2}+b^{2} \leqslant 2\left|q_{h}^{m}\right|^{2}+c \tau^{2} .
$$


Since $\frac{-1}{Q(u)}$ is the last component of $v(u)$ we obtain similarly as above

$$
\begin{aligned}
\left|S_{2}\right| & \leqslant c \int_{\Omega}\left(\left|q_{h}^{m}\right|^{2}+\tau^{2}\right)\left|v\left(u_{h}^{m}\right)-v\left(\hat{u}_{h}^{m}\right)\right| \\
& \leqslant c \gamma \int_{\Omega} \frac{\left(\left(Q\left(\hat{u}_{h}^{m+1}\right)-Q\left(u_{h}^{m+1}\right)\right)-\left(Q\left(\hat{u}_{h}^{m}\right)-Q\left(u_{h}^{m}\right)\right)\right)^{2}}{Q\left(u_{h}^{m}\right)}+c\left(\tau^{3}+\tau E^{m}\right) .
\end{aligned}
$$

Finally, note that

$$
\begin{aligned}
S_{3}+S_{4}= & \int_{\Omega}\left|v\left(\hat{u}_{h}^{m+1}\right)-v\left(u_{h}^{m+1}\right)\right|^{2} Q\left(u_{h}^{m+1}\right)\left(\frac{Q\left(\hat{u}_{h}^{m+1}\right)}{Q\left(u_{h}^{m}\right)}-1\right) \\
& +\int_{\Omega}\left|\left(v\left(\hat{u}_{h}^{m+1}\right)-v\left(u_{h}^{m+1}\right)\right)+\left(v\left(\hat{u}_{h}^{m}\right)-v\left(\hat{u}_{h}^{m+1}\right)\right)\right|^{2} Q\left(u_{h}^{m+1}\right)\left(1-\frac{Q\left(\hat{u}_{h}^{m}\right)}{Q\left(u_{h}^{m}\right)}\right) \\
= & \int_{\Omega}\left|v\left(\hat{u}_{h}^{m+1}\right)-v\left(u_{h}^{m+1}\right)\right|^{2} \frac{Q\left(u_{h}^{m+1}\right)}{Q\left(u_{h}^{m}\right)}\left(Q\left(\hat{u}_{h}^{m+1}\right)-Q\left(\hat{u}_{h}^{m}\right)\right) \\
& +\int_{\Omega}\left(2\left(v\left(\hat{u}_{h}^{m+1}\right)-v\left(u_{h}^{m+1}\right)\right) \cdot\left(v\left(\hat{u}_{h}^{m}\right)-v\left(\hat{u}_{h}^{m+1}\right)\right)\right. \\
& \left.+\left|v\left(\hat{u}_{h}^{m}\right)-v\left(\hat{u}_{h}^{m+1}\right)\right|^{2}\right) Q\left(u_{h}^{m+1}\right)\left(1-\frac{Q\left(\hat{u}_{h}^{m}\right)}{Q\left(u_{h}^{m}\right)}\right) .
\end{aligned}
$$

Thus, using similar arguments as in the estimate for $S_{1}$ we conclude

$$
\begin{aligned}
\left|S_{3}+S_{4}\right| & \leqslant c \tau E^{m+1}+c \tau \int_{\Omega}\left(\left|v\left(u_{h}^{m+1}\right)-v\left(\hat{u}_{h}^{m+1}\right)\right|+\tau\right)\left|v\left(u_{h}^{m}\right)-v\left(\hat{u}_{h}^{m}\right)\right| Q\left(u_{h}^{m+1}\right) \\
& \leqslant c \tau E^{m+1}+c \tau \int_{\Omega}\left(\left|v\left(u_{h}^{m+1}\right)-v\left(\hat{u}_{h}^{m+1}\right)\right|+\tau\right)\left|v\left(u_{h}^{m}\right)-v\left(\hat{u}_{h}^{m}\right)\right|\left(1+\left|q_{h}^{m}\right|\right) \\
& \leqslant \delta \int_{\Omega} \frac{\left(\left(Q\left(\hat{u}_{h}^{m+1}\right)-Q\left(u_{h}^{m+1}\right)\right)-\left(Q\left(\hat{u}_{h}^{m}\right)-Q\left(u_{h}^{m}\right)\right)\right)^{2}}{Q\left(u_{h}^{m}\right)}+\frac{c}{\delta}\left(\tau^{3}+\tau E^{m}+\tau E^{m+1}\right) .
\end{aligned}
$$

Combining (3.19) with the above estimates and choosing $\delta$ and $\gamma$ small enough completes the proof of the lemma.

Let us now return to (3.12) and estimate the four terms on the right-hand side of this relation.

(i) Since $\left\|\epsilon^{m+1}-\epsilon^{m}\right\| \leqslant c \tau$ sup $\left\|\epsilon_{t}\right\| \leqslant c \tau h^{2}|\log h|^{2}$ (by (3.7)) we obtain $(0, T)$

$$
\begin{aligned}
\frac{1}{\tau^{2}} \int_{\Omega} \frac{\left(\mathrm{e}^{m+1}-\mathrm{e}^{m}\right)\left(\epsilon^{m+1}-\epsilon^{m}\right)}{Q\left(u_{h}^{m}\right)} & \leqslant \frac{\delta}{\tau^{2}} \int_{\Omega} \frac{\left(\mathrm{e}^{m+1}-\mathrm{e}^{m}\right)^{2}}{Q\left(u_{h}^{m}\right)}+\frac{c}{\delta \tau^{2}}\left\|\epsilon^{m+1}-\epsilon^{m}\right\|^{2} \\
& \leqslant \frac{\delta}{\tau^{2}} \int_{\Omega} \frac{\left(\mathrm{e}^{m+1}-\mathrm{e}^{m}\right)^{2}}{Q\left(u_{h}^{m}\right)}+\frac{c}{\delta} h^{4}|\log h|^{4}
\end{aligned}
$$


(ii) Since $\mathrm{e}_{h}^{m+1}-\mathrm{e}_{h}^{m} \in X_{h 0}$, integration by parts yields

$$
\begin{aligned}
& \left|\frac{1}{\tau} \int_{\Omega} \frac{\nabla\left(\hat{u}_{h}^{m+1}-\hat{u}_{h}^{m}\right) \cdot \nabla\left(\mathrm{e}_{h}^{m+1}-\mathrm{e}_{h}^{m}\right)}{Q\left(\hat{u}_{h}^{m}\right)}\right|=\mid \frac{1}{\tau} \int_{\Omega} \frac{\nabla\left(u^{m+1}-u^{m}\right) \cdot \nabla\left(\mathrm{e}_{h}^{m+1}-\mathrm{e}_{h}^{m}\right)}{Q\left(u^{m}\right)} \\
& +\frac{1}{\tau} \int_{\Omega}\left(\frac{\nabla\left(\hat{u}_{h}^{m+1}-\hat{u}_{h}^{m}\right)}{Q\left(\hat{u}_{h}^{m}\right)}-\frac{\nabla\left(u^{m+1}-u^{m}\right)}{Q\left(u^{m}\right)}\right) \cdot \nabla\left(\mathrm{e}_{h}^{m+1}-\mathrm{e}_{h}^{m}\right) \mid \\
\leqslant & -\frac{1}{\tau} \int_{\Omega} \nabla \cdot\left(\frac{\nabla\left(u^{m+1}-u^{m}\right)}{Q\left(u^{m}\right)}\right)\left(\mathrm{e}_{h}^{m+1}-\mathrm{e}_{h}^{m}\right) \mid \\
& +\frac{c}{\tau} \int_{\Omega}\left(\frac{\left|\nabla\left(\epsilon^{m+1}-\epsilon^{m}\right)\right|}{Q\left(\hat{u}_{h}^{m}\right)}+\left|\nabla\left(u^{m+1}-u^{m}\right)\right|\left|\frac{1}{Q\left(u^{m}\right)}-\frac{1}{Q\left(\hat{u}_{h}^{m}\right)}\right|\right)\left|\nabla\left(\mathrm{e}_{h}^{m+1}-\mathrm{e}_{h}^{m}\right)\right| \\
\leqslant & \frac{c}{\tau}\left\|\nabla\left(u^{m+1}-u^{m}\right)\right\|_{H^{1,2}}\left\|\mathrm{e}_{h}^{m+1}-\mathrm{e}_{h}^{m}\right\| \\
& +\frac{c}{\tau}\left(\left\|\nabla\left(\epsilon^{m+1}-\epsilon^{m}\right)\right\|+\tau\left\|\nabla \epsilon^{m}\right\|\right)\left\|\nabla\left(\mathrm{e}_{h}^{m+1}-\mathrm{e}_{h}^{m}\right)\right\| \\
\leqslant & c\left(\left\|\mathrm{e}^{m+1}-\mathrm{e}^{m}\right\|+\left\|\epsilon^{m+1}-\epsilon^{m}\right\|\right)+c\left(\sup _{(0, T)}\left\|\nabla \epsilon_{t}\right\|+c h\right)\left\|\nabla\left(\mathrm{e}_{h}^{m+1}-\mathrm{e}_{h}^{m}\right)\right\| \\
\leqslant & \frac{\delta}{\tau} \int_{\Omega} \frac{\left|\nabla\left(\mathrm{e}_{h}^{m+1}-\mathrm{e}_{h}^{m}\right)\right|^{2}}{Q\left(\hat{u}_{h}^{m}\right)}+\frac{\delta}{\tau^{2}} \int_{\Omega} \frac{\left(\mathrm{e}^{m+1}-\mathrm{e}^{m}\right)^{2}}{Q\left(u_{h}^{m}\right)}+\frac{c}{\delta}\left(\tau^{2}+h^{4}|\log h|^{4}\right)
\end{aligned}
$$

where we used (3.5), (3.8) and once again (3.13).

Combining the above estimates we arrive at

$$
\begin{aligned}
& \left|\frac{1}{\tau} \int_{\Omega} \frac{\nabla\left(\hat{u}_{h}^{m+1}-\hat{u}_{h}^{m}\right) \cdot \nabla\left(\mathrm{e}_{h}^{m+1}-\mathrm{e}_{h}^{m}\right)}{Q\left(\hat{u}_{h}^{m}\right)}\right| \\
& \quad \leqslant \frac{\delta}{\tau} \int_{\Omega} \frac{\left|\nabla\left(\mathrm{e}_{h}^{m+1}-\mathrm{e}_{h}^{m}\right)\right|^{2}}{Q\left(\hat{u}_{h}^{m}\right)}+\frac{\delta}{\tau^{2}} \int_{\Omega} \frac{\left(\mathrm{e}^{m+1}-\mathrm{e}^{m}\right)^{2}}{Q\left(u_{h}^{m}\right)}+\frac{c}{\delta}\left(\tau^{2}+h^{4}|\log h|^{4}\right) .
\end{aligned}
$$

(iii) To begin, let us rewrite the difference $\frac{1}{Q\left(u_{h}^{m}\right)}-\frac{1}{Q\left(u^{m}\right)}$ as follows:

$$
\frac{1}{Q\left(u_{h}^{m}\right)}-\frac{1}{Q\left(u^{m}\right)}=-b_{h}^{m} \cdot \nabla \mathrm{e}^{m}
$$

where

$$
b_{h}^{m}=\int_{0}^{1} B\left(s \nabla u_{h}^{m}+(1-s) \nabla u^{m}\right) \mathrm{d} s, \quad B_{i}=\frac{\partial}{\partial p_{i}}\left(\frac{1}{\sqrt{1+|p|^{2}}}\right) .
$$


If we let in addition $b^{m}:=B\left(\nabla u^{m}\right)$ we may write

$$
\begin{aligned}
& \frac{1}{\tau^{2}} \int_{\Omega}\left(u^{m+1}-u^{m}\right)\left(\mathrm{e}_{h}^{m+1}-\mathrm{e}_{h}^{m}\right)\left(\frac{1}{Q\left(u_{h}^{m}\right)}-\frac{1}{Q\left(u^{m}\right)}\right) \\
= & -\frac{1}{\tau^{2}} \int_{\Omega}\left(u^{m+1}-u^{m}\right)\left(\mathrm{e}_{h}^{m+1}-\mathrm{e}_{h}^{m}\right) b_{h}^{m} \cdot \nabla \mathrm{e}_{h}^{m} \\
& -\frac{1}{\tau^{2}} \int_{\Omega}\left(u^{m+1}-u^{m}\right)\left(\mathrm{e}_{h}^{m+1}-\mathrm{e}_{h}^{m}\right) b^{m} \cdot \nabla \epsilon^{m} \\
& -\frac{1}{\tau^{2}} \int_{\Omega}\left(u^{m+1}-u^{m}\right)\left(\mathrm{e}_{h}^{m+1}-\mathrm{e}_{h}^{m}\right)\left(b_{h}^{m}-b^{m}\right) \cdot \nabla \epsilon^{m} \\
\equiv & I+I I+I I I .
\end{aligned}
$$

Since $\left|b_{h}^{m}\right| \leqslant 1$ uniformly in $h$ and $m$ we obtain for $0<\delta \leqslant 1$ with the help of (3.14)

$$
\begin{aligned}
|I| & \leqslant \frac{c}{\tau} \int_{\Omega}\left|\mathrm{e}_{h}^{m+1}-\mathrm{e}_{h}^{m}\right|\left|\nabla \mathrm{e}_{h}^{m}\right| \leqslant \frac{\delta}{\tau^{2}} \int_{\Omega} \frac{\left|\mathrm{e}_{h}^{m+1}-\mathrm{e}_{h}^{m}\right|^{2}}{Q\left(u_{h}^{m}\right)}+\frac{c}{\delta}\left\|\nabla \mathrm{e}_{h}^{m}\right\|^{2} \\
& \leqslant \frac{2 \delta}{\tau^{2}} \int_{\Omega} \frac{\left|\mathrm{e}^{m+1}-\mathrm{e}^{m}\right|^{2}}{Q\left(u_{h}^{m}\right)}+\frac{c}{\tau^{2}}\left\|\epsilon^{m+1}-\epsilon^{m}\right\|^{2}+\frac{c}{\delta} E^{m} \\
& \leqslant \frac{2 \delta}{\tau^{2}} \int_{\Omega} \frac{\left|\mathrm{e}^{m+1}-\mathrm{e}^{m}\right|^{2}}{Q\left(u_{h}^{m}\right)}+c h^{4}|\log h|^{4}+\frac{c}{\delta} E^{m} .
\end{aligned}
$$

Next, as $\left|b_{h}^{m}-b^{m}\right| \leqslant c\left|\nabla \mathrm{e}^{m}\right|$ we may estimate

$$
\begin{aligned}
|I I I| & \leqslant \frac{c}{\tau}\left\|\nabla \epsilon^{m}\right\|_{L^{\infty}} \int_{\Omega}\left|\mathrm{e}_{h}^{m+1}-\mathrm{e}_{h}^{m}\right|\left|\nabla \mathrm{e}^{m}\right| \\
& \leqslant \frac{\delta}{\tau^{2}} \int_{\Omega} \frac{\left|\mathrm{e}_{h}^{m+1}-\mathrm{e}_{h}^{m}\right|^{2}}{Q\left(u_{h}^{m}\right)}+\frac{c}{\delta}\left\|\nabla \epsilon^{m}\right\|_{L^{\infty}}^{2}\left(\left\|\nabla \epsilon^{m}\right\|^{2}+\left\|\nabla \mathrm{e}_{h}^{m}\right\|^{2}\right) \\
& \leqslant \frac{2 \delta}{\tau^{2}} \int_{\Omega} \frac{\left|\mathrm{e}^{m+1}-\mathrm{e}^{m}\right|^{2}}{Q\left(u_{h}^{m}\right)}+\frac{c}{\delta} h^{4}|\log h|^{4}+\frac{c}{\delta} E^{m} .
\end{aligned}
$$

It remains to examine $I I$. Integration by parts gives

$$
\begin{aligned}
I I=\frac{1}{\tau^{2}} \int_{\Omega} b^{m} \cdot \nabla\left(u^{m+1}-u^{m}\right)\left(\mathrm{e}_{h}^{m+1}-\mathrm{e}_{h}^{m}\right) \epsilon^{m} \\
\quad+\frac{1}{\tau^{2}} \int_{\Omega} b^{m} \cdot \nabla\left(\mathrm{e}_{h}^{m+1}-\mathrm{e}_{h}^{m}\right)\left(u^{m+1}-u^{m}\right) \epsilon^{m} \\
\quad+\frac{1}{\tau^{2}} \int_{\Omega} \nabla \cdot b^{m}\left(u^{m+1}-u^{m}\right)\left(\mathrm{e}_{h}^{m+1}-\mathrm{e}_{h}^{m}\right) \epsilon^{m} \\
\equiv I I_{1}+I I_{2}+I I_{3} .
\end{aligned}
$$

Using the regularity of $u$ and similar arguments as above we get

$$
\left|I I_{1}\right|+\left|I I_{3}\right| \leqslant \frac{c}{\tau} \int_{\Omega}\left|\mathrm{e}_{h}^{m+1}-\mathrm{e}_{h}^{m}\right|\left|\epsilon^{m}\right| \leqslant \frac{2 \delta}{\tau^{2}} \int_{\Omega} \frac{\left|\mathrm{e}^{m+1}-\mathrm{e}^{m}\right|^{2}}{Q\left(u_{h}^{m}\right)}+\frac{c}{\delta} h^{4} .
$$


Observing that $u^{m+1}-u^{m}=\tau u_{t}\left(\cdot, t_{m}\right)+R^{m}$ (where $R^{m}$ satisfies (3.10)) and letting $d^{m}:=$ $u_{t}\left(\cdot, t_{m}\right) b^{m}$, the remaining term $I I_{2}$ can be rewritten as

$$
\begin{aligned}
I I_{2}= & \frac{1}{\tau} \int_{\Omega} d^{m} \cdot \nabla\left(\mathrm{e}_{h}^{m+1}-\mathrm{e}_{h}^{m}\right) \epsilon^{m}+\frac{1}{\tau^{2}} \int_{\Omega} b^{m} \cdot \nabla\left(\mathrm{e}_{h}^{m+1}-\mathrm{e}_{h}^{m}\right) R^{m} \epsilon^{m} \\
= & \frac{1}{\tau} \int_{\Omega} d^{m+1} \cdot \nabla \mathrm{e}_{h}^{m+1} \epsilon^{m+1}-\frac{1}{\tau} \int_{\Omega} d^{m} \cdot \nabla \mathrm{e}_{h}^{m} \epsilon^{m}-\frac{1}{\tau} \int_{\Omega}\left(d^{m+1}-d^{m}\right) \cdot \nabla \mathrm{e}_{h}^{m+1} \epsilon^{m+1} \\
& \quad-\frac{1}{\tau} \int_{\Omega} d^{m} \cdot \nabla \mathrm{e}_{h}^{m+1}\left(\epsilon^{m+1}-\epsilon^{m}\right)+\frac{1}{\tau^{2}} \int_{\Omega} b^{m} \cdot \nabla\left(\mathrm{e}_{h}^{m+1}-\mathrm{e}_{h}^{m}\right) R^{m} \epsilon^{m} .
\end{aligned}
$$

We shall keep the discrete time derivative and estimate the last three integrals. To begin, note that

$$
\begin{aligned}
d^{m+1}-d^{m} & =u_{t}\left(\cdot, t_{m+1}\right) B\left(\nabla u^{m+1}\right)-u_{t}\left(\cdot, t_{m}\right) B\left(\nabla u^{m}\right) \\
& =\int_{t_{m}}^{t_{m+1}} u_{t t}(\cdot, s) \mathrm{d} s B\left(\nabla u^{m+1}\right)+u_{t}\left(\cdot, t_{m}\right) \int_{t_{m}}^{t_{m+1}} B^{\prime}(\nabla u(\cdot, s)) \nabla u_{t}(\cdot, s) \mathrm{d} s .
\end{aligned}
$$

Recalling that $u_{t}, \nabla u_{t} \in L^{\infty}(\Omega \times(0, T))$ we infer

$$
\left|d^{m+1}-d^{m}\right| \leqslant c\left|\int_{t_{m}}^{t_{m+1}} u_{t t}(\cdot, s) \mathrm{d} s\right|+c \tau,
$$

so that (3.5) and (3.6) imply

$$
\begin{aligned}
& \left|-\frac{1}{\tau} \int_{\Omega}\left(d^{m+1}-d^{m}\right) \cdot \nabla \mathrm{e}_{h}^{m+1} \epsilon^{m+1}\right| \\
\leqslant & \frac{c}{\tau}\left(\left\|\epsilon^{m+1}\right\|_{L^{\infty}}\left\|\int_{t_{m}}^{t_{m+1}} u_{t t}(., s) \mathrm{d} s\right\|+\tau\left\|\epsilon^{m+1}\right\|\right)\left\|\nabla \mathrm{e}_{h}^{m+1}\right\| \\
\leqslant & c\left(\sup _{(0, T)}\left\|u_{t t}\right\|\left\|\epsilon^{m+1}\right\|_{L^{\infty}}+\left\|\epsilon^{m+1}\right\|\right)\left\|\nabla \mathrm{e}_{h}^{m+1}\right\| \\
\leqslant & c h^{2}|\log h|\left(\left\|\nabla\left(\mathrm{e}_{h}^{m+1}-\mathrm{e}_{h}^{m}\right)\right\|+\left\|\nabla \mathrm{e}_{h}^{m}\right\|\right) \\
\leqslant & \frac{\delta}{\tau} \int_{\Omega} \frac{\left|\nabla\left(\mathrm{e}_{h}^{m+1}-\mathrm{e}_{h}^{m}\right)\right|^{2}}{Q\left(\hat{u}_{h}^{m}\right)}+c E^{m}+\frac{c}{\delta} h^{4}(\log h)^{2} .
\end{aligned}
$$

Furthermore, using (3.10) and (3.6) we have

$$
\begin{aligned}
\mid- & \frac{1}{\tau} \int_{\Omega} d^{m} \cdot \nabla \mathrm{e}_{h}^{m+1}\left(\epsilon^{m+1}-\epsilon^{m}\right)+\frac{1}{\tau^{2}} \int_{\Omega} b^{m} \cdot \nabla\left(\mathrm{e}_{h}^{m+1}-\mathrm{e}_{h}^{m}\right) R^{m} \epsilon^{m} \mid \\
& \leqslant \frac{c}{\tau} \int_{\Omega}\left(\left|\nabla \mathrm{e}_{h}^{m}\right|+\left|\nabla\left(\mathrm{e}_{h}^{m+1}-\mathrm{e}_{h}^{m}\right)\right|\right)\left|\epsilon^{m+1}-\epsilon^{m}\right|+\frac{c}{\tau^{2}} \int_{\Omega}\left|\nabla\left(\mathrm{e}_{h}^{m+1}-\mathrm{e}_{h}^{m}\right)\right|\left|R^{m}\right|\left|\epsilon^{m}\right| \\
& \leqslant \frac{c}{\tau}\left\|\epsilon^{m+1}-\epsilon^{m}\right\|\left(\left\|\nabla \mathrm{e}_{h}^{m}\right\|+\left\|\nabla\left(\mathrm{e}_{h}^{m+1}-\mathrm{e}_{h}^{m}\right)\right\|\right)+c\left\|\nabla\left(\mathrm{e}_{h}^{m+1}-\mathrm{e}_{h}^{m}\right)\right\|\left\|\epsilon^{m}\right\|_{L^{\infty}} \\
& \leqslant \frac{\delta}{\tau} \int_{\Omega} \frac{\left|\nabla\left(\mathrm{e}_{h}^{m+1}-\mathrm{e}_{h}^{m}\right)\right|^{2}}{Q\left(\hat{u}_{h}^{m}\right)}+\frac{c}{\delta} h^{4}|\log h|^{4}+c E^{m} .
\end{aligned}
$$


Collating our estimates we finally get

$$
\begin{aligned}
& \frac{1}{\tau^{2}} \int_{\Omega}\left(u^{m+1}-u^{m}\right)\left(\mathrm{e}_{h}^{m+1}-\mathrm{e}_{h}^{m}\right)\left(\frac{1}{Q\left(u_{h}^{m}\right)}-\frac{1}{Q\left(u^{m}\right)}\right) \\
& \leqslant \frac{1}{\tau} \int_{\Omega} d^{m+1} \cdot \nabla \mathrm{e}_{h}^{m+1} \epsilon^{m+1}-\frac{1}{\tau} \int_{\Omega} d^{m} \cdot \nabla \mathrm{e}_{h}^{m} \epsilon^{m} \\
&+\frac{6 \delta}{\tau^{2}} \int_{\Omega} \frac{\left|\mathrm{e}^{m+1}-\mathrm{e}^{m}\right|^{2}}{Q\left(u_{h}^{m}\right)}+\frac{2 \delta}{\tau} \int_{\Omega} \frac{\left|\nabla\left(\mathrm{e}_{h}^{m+1}-\mathrm{e}_{h}^{m}\right)\right|^{2}}{Q\left(\hat{u}_{h}^{m}\right)}+\frac{c}{\delta} h^{4}|\log h|^{4}+\frac{c}{\delta}\left(E^{m}+E^{m+1}\right) .
\end{aligned}
$$

(iv) From (3.10) and (3.13) we obtain

$$
\begin{aligned}
\left|\frac{1}{\tau^{2}} \int_{\Omega} \frac{R^{m}\left(\mathrm{e}_{h}^{m+1}-\mathrm{e}_{h}^{m}\right)}{Q\left(u^{m}\right)}\right| & \leqslant c\left\|\mathrm{e}_{h}^{m+1}-\mathrm{e}_{h}^{m}\right\| \leqslant c\left(\left\|\mathrm{e}^{m+1}-\mathrm{e}^{m}\right\|+\left\|\epsilon^{m+1}-\epsilon^{m}\right\|\right) \\
& \leqslant \frac{\delta}{\tau^{2}} \int_{\Omega} \frac{\left(\mathrm{e}^{m+1}-\mathrm{e}^{m}\right)^{2}}{Q\left(u_{h}^{m}\right)}+c \frac{\tau^{2}}{\delta}+c \tau h^{2}|\log h|^{2} .
\end{aligned}
$$

If we apply Lemma 2 and our estimates (i)-(iv) to (3.12) we obtain, by choosing $\delta$ sufficiently small,

$$
\begin{aligned}
& \frac{1}{2 \tau^{2}} \int_{\Omega} \frac{\left|\mathrm{e}^{m+1}-\mathrm{e}^{m}\right|^{2}}{Q\left(u_{h}^{m}\right)}+\frac{1}{8 \tau} \int_{\Omega} \frac{\left|\nabla\left(\mathrm{e}_{h}^{m+1}-\mathrm{e}_{h}^{m}\right)\right|^{2}}{Q\left(\hat{u}_{h}^{m}\right)}+\frac{1}{2 \tau}\left(E^{m+1}-E^{m}\right) \\
& \quad \leqslant \frac{1}{\tau} \int_{\Omega} d^{m+1} \cdot \nabla \mathrm{e}_{h}^{m+1} \epsilon^{m+1}-\frac{1}{\tau} \int_{\Omega} d^{m} \cdot \nabla \mathrm{e}_{h}^{m} \epsilon^{m}+c\left(E^{m}+E^{m+1}\right)+c\left(\tau^{2}+h^{4}|\log h|^{4}\right) .
\end{aligned}
$$

Now we are in position to complete the proof of Theorem 1. In a first step we claim that

$$
h^{-2} E^{m} \leqslant \gamma^{2} \quad \text { for all } \quad 0 \leqslant m \leqslant M
$$

provided $\tau \leqslant \delta_{0} h, h \leqslant h_{0}$ and $\gamma$ is chosen according to Lemmas 1 and 2 . Since $E^{0}=0$, the assertion clearly holds for $m=0$. Assuming that (3.26) is true for $k \leqslant m$ we may multiply (3.25) (with $m$ replaced by $j$ ) by $\tau$ and sum from $j=0, \ldots, k$ :

$$
\begin{aligned}
\frac{1}{2} E^{k+1} & \leqslant \int_{\Omega} d^{k+1} \cdot \nabla \mathrm{e}_{h}^{k+1} \epsilon^{k+1}+c \tau \sum_{j=0}^{k}\left(E^{j}+E^{j+1}\right)+c\left(\tau^{2}+h^{4}|\log h|^{4}\right) \\
& \leqslant\left(\frac{1}{4}+\tau\right) E^{k+1}+c \tau \sum_{j=0}^{k} E^{j}+c\left(\tau^{2}+h^{4}|\log h|^{4}\right) .
\end{aligned}
$$

Here, we used the inequality

$$
\left|\int_{\Omega} d^{k+1} \cdot \nabla \mathrm{e}_{h}^{k+1} \epsilon^{k+1}\right| \leqslant \frac{1}{4} E^{k+1}+c h^{4}|\log h|^{2}
$$

which can be derived in essentially the same way as (3.24). If $\tau$ is small enough we obtain

$$
E^{k+1} \leqslant c \tau \sum_{j=0}^{k} E^{j}+c\left(\tau^{2}+h^{4}|\log h|^{4}\right), \quad 0 \leqslant k \leqslant m
$$


and a discrete Gronwall argument implies

$$
E^{k+1} \leqslant c\left(\tau^{2}+h^{4}|\log h|^{4}\right), \quad 0 \leqslant k \leqslant m .
$$

In particular,

$$
E^{m+1} \leqslant c\left(\tau^{2}+h^{4}|\log h|^{4}\right) \leqslant c\left(\delta_{0}^{2} h^{2}+h^{4}|\log h|^{4}\right) \leqslant \gamma^{2} h^{2}
$$

provided $\tau \leqslant \delta_{0} h, h \leqslant h_{0}$ and $\delta_{0}$ is chosen sufficiently small. Thus, (3.26) is proved and we can start again from (3.25) in order to show that

$$
E^{k} \leqslant c\left(\tau^{2}+h^{4}|\log h|^{4}\right) \quad \text { for all } \quad 0 \leqslant k \leqslant M
$$

as well as

$$
\sum_{m=0}^{M-1} \frac{1}{\tau} \int_{\Omega} \frac{\left|\mathrm{e}^{m+1}-\mathrm{e}^{m}\right|^{2}}{Q\left(u_{h}^{m}\right)} \leqslant c\left(\tau^{2}+h^{4}|\log h|^{4}\right) .
$$

Since $Q\left(u_{h}^{m}\right)$ is uniformly bounded, this implies

$$
\begin{aligned}
\left\|\mathrm{e}^{k}\right\| & \leqslant\left\|\mathrm{e}^{0}\right\|+\sum_{m=0}^{k-1}\left\|\mathrm{e}^{m+1}-\mathrm{e}^{m}\right\| \leqslant c h^{2}+\left(\frac{1}{\tau} \sum_{m=0}^{M-1}\left\|\mathrm{e}^{m+1}-\mathrm{e}^{m}\right\|^{2}\right)^{\frac{1}{2}} \\
& \leqslant c\left(\tau+h^{2}|\log h|^{2}\right) .
\end{aligned}
$$

Finally,

$$
\left\|\nabla\left(u^{m}-u_{h}^{m}\right)\right\| \leqslant\left\|\nabla \mathrm{e}_{h}^{m}\right\|+\left\|\nabla \epsilon^{m}\right\| \leqslant c\left(\sqrt{E^{m}}+h\right) \leqslant c(\tau+h)
$$

by (3.5) and (3.14). This completes the proof of Theorem 1.

\section{Implementation and numerical tests}

The scheme (2.2) is such that in every time step a linear system of equations has to be solved. Assume that $X_{h}=\operatorname{span}\left\{\varphi_{1}, \ldots, \varphi_{N}\right\}$ with the nodal basis functions $\varphi_{j}(j=1, \ldots, N)$. Then $u_{h}^{m}$ has the form $u_{h}^{m}(x)=\sum_{j=1}^{N} u_{j}^{m} \varphi_{j}(x)$ with coefficients $\underline{u}^{m}=\left(u_{1}^{m}, \ldots, u_{N}^{m}\right)$. For given $u_{h}^{m-1}$ the linear system then is

$$
\left(\frac{1}{\tau} M^{m-1}+S^{m-1}\right) \underline{u}^{m}=\frac{1}{\tau} M^{m-1} \underline{u}^{m-1}
$$

with mass matrix $M^{m-1}$ and stiffness matrix $S^{m-1}$

$$
M_{i j}^{m-1}=\int_{\Omega} \frac{\varphi_{i} \varphi_{j}}{Q\left(u_{h}^{m-1}\right)}, \quad S_{i j}^{m-1}=\int_{\Omega} \frac{\nabla \varphi_{i} \nabla \varphi_{j}}{Q\left(u_{h}^{m-1}\right)}, \quad(i, j=1, \ldots, N) .
$$

The matrices are symmetric and $M^{m-1} / \tau+S^{m-1}$ is positive definite and we can use a suitable conjugate gradient algorithm to solve the linear system.

We are going to verify the asymptotic error estimates of the Theorem. For this we use an exact solution of the equation for the mean curvature flow of graphs with a given right-hand side, i.e. with 
TABLE 1

Absolute errors in $L^{\infty}\left((0, T) ; L^{2}(\Omega)\right)$ and experimental orders of convergence (EOC) for the test problem

\begin{tabular}{lllll}
\hline \multicolumn{1}{c}{$h$} & \multicolumn{1}{c}{$\tau=h^{2}$} & \multicolumn{1}{c}{$\tau=0.25 h$} & \multicolumn{1}{c}{$\tau=0.5 h$} & \multicolumn{1}{c}{$\tau=h$} \\
\hline 2.0 & 1.1608 & 1.1925 & 1.1608 & 1.1608 \\
1.0 & $0.6903(0.75)$ & $0.6732(0.82)$ & $0.6671(0.80)$ & $0.6903(0.75)$ \\
0.7368 & $0.4141(1.67)$ & $0.3119(2.52)$ & $0.3584(2.03)$ & $0.4480(1.42)$ \\
0.4203 & $0.1836(1.45)$ & $0.1416(1.41)$ & $0.1995(1.04)$ & $0.2851(0.81)$ \\
0.2219 & $0.0600(1.75)$ & $0.06510(1.22)$ & $0.1065(0.98)$ & $0.1723(0.79)$ \\
0.1137 & $0.01656(1.93)$ & $0.03061(1.13)$ & $0.05490(0.99)$ & $0.09827(0.84)$ \\
0.05754 & $0.004285(1.99)$ & $0.01447(1.10)$ & $0.02787(0.99)$ & $0.05260(0.92)$ \\
\hline
\end{tabular}

TABLE 2

Absolute errors in $L^{\infty}\left((0, T) ; H^{1}(\Omega)\right)$ and experimental orders of convergence $(E O C)$ for the test problem

\begin{tabular}{lllll}
\hline \multicolumn{1}{c}{$h$} & \multicolumn{1}{c}{$\tau=h^{2}$} & \multicolumn{1}{c}{$\tau=0.25 h$} & \multicolumn{1}{c}{$\tau=0.5 h$} & \multicolumn{1}{c}{$\tau=h$} \\
\hline 2.0 & 0.6916 & 0.9141 & 0.9129 & 0.6916 \\
1.0 & $1.0025(-0.54)$ & $0.9755(-0.09)$ & $0.7169(0.35)$ & $1.0025(-0.54)$ \\
0.7368 & $0.8899(0.39)$ & $0.6253(1.46)$ & $0.8013(-0.36)$ & $0.9913(0.04)$ \\
0.4203 & $0.4708(1.13)$ & $0.3676(0.95)$ & $0.5124(0.80)$ & $0.6596(0.73)$ \\
0.2219 & $0.1786(1.52)$ & $0.1912(1.02)$ & $0.2991(0.84)$ & $0.4766(0.51)$ \\
0.1137 & $0.06301(1.56)$ & $0.09666(1.02)$ & $0.1634(0.90)$ & $0.2834(0.78)$ \\
0.05754 & $0.02628(1.28)$ & $0.04844(1.01)$ & $0.0856(0.95)$ & $0.1568(0.87)$ \\
\hline
\end{tabular}

prescribed mean curvature. This introduces additional error terms in the analysis not presented in this paper. We solve the geometric equation

$$
u_{t}-Q(u) \nabla \cdot \frac{\nabla u}{Q(u)}=f Q(u)
$$

on the unit disk $\Omega=\left\{x \in \mathbb{R}^{2}|| x \mid<1\right\}$ with zero boundary conditions $u=0$ on $\partial \Omega$. The solution is given by

$$
u(x, t)=\sin \left(|x|^{2}-t\right)-\sin (1-t) .
$$

The solution does not decay for large time $t$ and the norm $L^{\infty}\left((0, T) ; H^{1}(\Omega)\right)$ is quite large. We have chosen $T=4$ for the test computations. The right-hand side is calculated from equation (4.1) and then used in the program to compute the discrete solution. For two sucessive grids with grid sizes $h_{1}$ and $h_{2}$ we computed the absolute errors $\operatorname{Err}\left(h_{j}\right),(j=1,2)$ between discrete solution and exact solution for certain norms. The experimental order of convergence then was defined by

$$
\operatorname{EOC}\left(h_{1}, h_{2}\right)=\frac{\log \frac{\operatorname{Err}\left(h_{1}\right)}{\operatorname{Err}\left(h_{2}\right)}}{\log \frac{h_{1}}{h_{2}}} .
$$

In Table $1 \operatorname{Err}(h)=\sup _{0 \leqslant m \leqslant M}\left\|u^{m}-u_{h}^{m}\right\|$ with $M \tau=T$ and in Table $2 \operatorname{Err}(h)=$ $\sup _{0 \leqslant m \leqslant M}\left\|\nabla\left(u^{m}-u_{h}^{m}\right)\right\|$. We see that the computations confirm the results of the Theorem precisely. In order to demonstrate the dependency on the coupling parameter $\delta_{0}$ in the condition $\tau \leqslant \delta_{0} h$ we provide computations for $\delta_{0}=h, 0.25,0.5$ and 1.0. 


\section{Acknowledgements}

This work was supported by the German Science Foundation (DFG) via Graduiertenkolleg Nichtlineare Differentialgleichungen: Modellierung, Theorie, Numerik, Visualisierung.

\section{REFERENCES}

1. Altschuler, S. J. \& Wu, L.-F. Convergence to translating solutions for a class of quasi-linear parabolic boundary problems. Math. Ann. 295, (1993) 761-765.

2. Altschuler, S. J. \& WU, L.-F. Translating surfaces of the non-parametric mean curvature flow with prescribed contact angle. Calc. Var. 2, (1994) 101-111.

3. Chen, X., Elliott, C. M., Gardiner, A., \& ZhaO, J. J. Convergence of numerical solutions to the Allen-Cahn equation. Appl. Anal. 69, (1998) 47-56.

4. Crandall, M. G. \& Lions, P. L. Convergent difference schemes for non-linear parabolic equations and mean curvature motion. Numer. Math. 75, (1996) 17-41.

5. DECKELNICK, K. Error analysis for a difference scheme approximating mean curvature flow. Interfaces and Free Boundaries 2, (2000) 117-142.

6. Deckelnick, K. \& DziUK, G. Convergence of a finite element method for non-parametric mean curvature flow. Numer. Math. 72, (1995) 197-222.

7. Deckelnick, K. \& DZIUK, G. Discrete anisotropic curvature flow of graphs. Math. Modelling Numer. Anal. 33, (1999) 1203-1222.

8. DZIUK, G. Numerical schemes for the mean curvature flow of graphs. In: Argoul, P., Frémond, M. \& NGUYEN, Q. S. (eds), IUTAM Symposium on Variations of Domains and Free-Boundary Problems in Solid Mechanics. pp. 63-70. Kluwer Academic Publishers, Dordrecht (1999).

9. Huisken, G. Non-parametric mean curvature evolution with boundary conditions. J. Diff. Eqns 77, (1989) 369-378.

10. Johnson, C. \& Thomée, V. Error estimates for a finite element approximation of a minimal surface. Math. Comput. 29, (1975) 343-349.

11. Lieberman, G. The first initial-boundary value problem for quasi-linear second-order parabolic equations. Ann. Scuola Norm. Sup. Pisa 13, (1986) 347-387.

12. Nochetto, R. H. \& Verdi, C. Convergence past singularities for a fully discrete approximation of curvature driven interfaces. SIAM J. Numer. Anal. 34, (1997) 490-512.

13. Oliker, V. I. \& URAltseva, N. N. Evolution of non-parametric surfaces with speed depending on curvature II. The mean curvature case. Commun. Pure Appl. Math. 46, (1993) 97-135.

14. Osher, S. \& SeTHIAN, J. A. Fronts propagating with curvature dependent speed: algorithms based on Hamilton-Jacobi formulations. J. Comput. Phys. 79, (1988) 12-49.

15. Walkington, N. J. Algorithms for computing motion by mean curvature. SIAM J. Numer. Anal. 33, (1996) 2215-2238.

16. Zlamal, M. Curved elements in the finite element method. Part I. SIAM J. Numer. Anal. 10, (1973) 229-240 Part II. SIAM J. Numer. Anal. 11, (1974) 347-362. 